\title{
Effect of vigorous physical activity on blood lipid and glucose
}

\author{
Hyoung-Jeong Kwon, Han-Joon Lee* \\ School of Exercise \& Sport Science, College of Natural Sciences, University of Ulsan, Ulsan, Korea
}

The aim of the study is to investigate how the participation of vigorous physical activities in the health examination contributes to blood lipid and blood glucose. A total of 56,810 workers from the Ulsan University Hospital in Ulsan, Subjects were tested for health checkups from February to November in 2016. The subject is those who does not have medical history, current ailments, and medication histories, and selected those who conducted the study of subjects tested to research. And this study did not consider their drinking and smoking. The final selected population was 11,557 and categorized as a vigorous physical activity of the health survey items. In this study, the group participated by the vigorous physical activity activities, group $1(n=70)$ had more than 6 days of vigorous physical activity, group $2(n=2,960)$ is 3 to 5 days of vigorous physical activity, the group $3(n=7,389)$ is 1 to 2 days of vigorous physical activity. The group $4(n=1,138)$ were classified as those who did not perform vigorous physical activity. To achieve the purpose of the study, the questionnaire examined blood lipid and blood glucose, using questions related to physical activity related to health examination in the Ulsan University Hospital. We obtained the mean and standard deviation for each group and conducted the one-way analysis of variance as an independent variable. Post hoc is least significant difference test and significant level is 0.05 . Vigorous physical activity more than 3 days of participation had a positive affect high-density lipoprotein cholesterol and triglyceride. But participation in vigorous physical activity did not affect blood glucose.

Keywords: Vigorous, Physical activity, Blood lipid, Blood glucose, Health questionnaire

\section{INTRODUCTION}

These days when the words 'the times of 100 years of age' are familiar, everyone should check his usual lifestyle to see whether he is ready to greet a healthy old age. Health is the most important part of old age in the age of healthy longevity. Health is the most important part of old age. Participant in regular exercise and physical activity has been steadily increasing with a view to enhancing satisfaction in complex tasks at work places and routine (Kim et al., 2011). The benefits that can be obtained through regular exercise and physical activities can be said to be innumerable. Regular exercise and physical activities are said to well treat the stress coming from limited life, provide mental and physical health and pleasure, strengthen the heart muscles, increase blood flows to the heart, reduce blood fats, maintain normal blood pres- sure and body weight, and increase the quantity of high-density lipoprotein cholesterol (HDL-C), while reducing the quantity of low-density lipoprotein cholesterol (LDL-C) (Lee et al., 2010). In addition, exercise is known to lower blood glucose and bring about useful changes in cardiovascular disease risk factors such as obesity and hypertension leading to further increases in interest in exercise and physical activities (Hermansen and Stensvold, 1972).

The American College of Sports Medicine (ACSM) and the American Heart Association reported that physical activities recommended to all healthy adults aged 18-65 years are moderate intensity aerobic physical activities with heart rates in a range of $50 \%-60 \%$ of the maximum heart rate for at least $40-60 \mathrm{~min} /$ day for at least 5 days/week or high intensity physical activities such as resistance exercise and jogging for at least $20 \mathrm{~min} /$ day for at least 3 days/week and that the recommendations can be met only
${ }^{*}$ Corresponding author: Han-Joon Lee (i] https://orcid.org/0000-0003-1109-3786 School of Exercise \& Sport Science, College of Natural Sciences, University of Ulsan, 93 Daehak-ro, Nam-gu, Ulsan 44610, Korea

Tel: +82-52-259-2384, Fax: +82-52-259-1696, E-mail: hanjoon@ulsan.ac.kr Received: November 1, 2017 / Accepted: December 9, 2017
This is an Open Access article distributed under the terms of the Creative Commons Attribution Non-Commercial License (http://creativecommons.org/licenses/by-nc/4.0/) which permits unrestricted non-commercial use, distribution, and reproduction in any medium, provided the original work is properly cited. 
when moderate intensity exercise and high intensity exercise are performed in combination (ACSM, 2014). In recent years, along with such guidelines, the effects of high intensity and vigorous exercises as well as those of moderate intensity exercise have been identified and recommended in many cases. Baker et al. (1986) reported a change in exercise intensity of $60 \%-80 \%$ during 20 weeks of aerobic exercise. They reported a significant reduction in LDL-C in men at $72 \%$ exercise intensity, suggesting that the reduction in LDL-C affects food and high-intensity exercise. In addition, studies of LDL-C according to exercise intensity reported no significant reduction in exercise intensity below $45 \%$ (Gaesser and Rich, 1984), and it was reported that high-intensity exercise and continuous exercise lowered LDL-C (Brownell et al., 1982). In a study conducted Jeong et al. (2014), the researchers reported that the ratio of those that showed hyperlipidemia was $8.8 \%$ among those that were performing vigorous physical activities while being $12.2 \%$ among those that were not performing vigorous exercise and that the ratio of LDL-C/HDL-C was significantly lower in the group of those that were performing vigorous exercise.

Impaired glucose tolerance is a stage prior to diabetes, when insulin resistance characteristically appears due to obesity and physical inactivity (Twigg et al., 2007). The clinical significance of impaired glucose tolerance is that although diabetes has not yet occurred, the risk of cardiovascular disease is higher compared to normal glucose tolerance due to insulin resistance (Rasmussen et al., 2008). Regular exercise is an effective way to prevent the impaired glucose tolerance as such from progressing into diabetes. The risk of diabetes is reduced by $30 \%-40 \%$ with participation in exercise once per week, and participation in exercise one time may improve insulin sensitivity and blood glucose for 24-72 hr (Colberg et al., 2010). According to previous studies on the improvement of blood glucose in middle-aged women through exercise, among different exercise programs, interval exercise showed significantly more improvements than continuous aerobic exercise 2-3 hr after completion of exercise.

Although previous studies examined exercise and biological variables as mentioned above, most of studies on the effects of exercise examined the effects of moderate intensity exercise and few studies analyzed the effects of high intensity and strenuous activities. Therefore, the present study aims to examine the effects of the frequency of participation in vigorous activities on blood lipids and blood glucose in the subjects of health examination survey in Ulsan University Hospital that various variables would change differently depending on how often the subjects participated in vigorous physical activities.

\section{MATERIALS AND METHODS}

\section{Design and subjects}

The present study was conducted with 56,810 workers out of those that underwent health examination survey at Ulsan University Hospital located in Ulsan Metropolitan City between February and November 2016. From among the subjects, those who did not have any past disease history, current disease, or history of medication were extracted and those that underwent the tests of those items that were to be studied. Whether the subjects were smoking or drinking was not considered. The number of subjects in the finally selected population was 11,557 and the subjects were classified according to strenuous physical activities among the health check-up interview items.

Of the 11,557 subjects in the population, 11,553 were production workers accounting for $99.9 \%$ of the population and of the production workers, 9,823 (85\%) were production workers in the shipbuilding industry.

In the present study, those subjects that were participating in vigorous physical activities for at least 6 days per week into group 1 , those that were participating in vigorous physical activities for 3-5 days into group 2, those that were participating in vigorous physical activities for 1-2 days into group 3, and those that were not participating in any vigorous physical activity into group 4 . To control those subjects that responded to the questionnaire unfaithfully, other subjects who responded that they did not participate in vigorous physical activities, moderate activities, or mild activities were excluded from the analysis. Out of the selected subjects in the population, 70 were assigned to group 1, 2,960 to group 2, 7,389 to group 3, and 1,138 to group 4. The characteristics of subjects were summarized in Table 1 .

\section{Questionnaire}

Physical activities related questions in the common questionnaire for health examination survey used by Ulsan University Hospital were used. The contents of the questions were; "During the last one week, on how many days did you perform vigorous activities that make you be out of breath much more than ordinary days for at least 20 min per day? (e.g., running, aerobics, fast biking, mountain climbing, etc.)." Those that participated in vigorous physical activities for at least 6 days were assigned to group 1 , those that participated in strenuous physical activities for 3-5 days to group 2, those that participated in strenuous physical activities for 1-2 days to group 3, and those that did not perform any strenuous physical activity to group 4 . 
Table 1. Characteristic of subjects

\begin{tabular}{lcccccc}
\hline Characteristic & Group 1 & Group 2 & Group 3 & Group 4 & $F$ & Post hoc \\
\hline Age $(\mathrm{yr})$ & $44.47 \pm 12.93$ & $42.97 \pm 11.01$ & $42.30 \pm 10.72$ & $43.10 \pm 10.06$ & $17.881^{* *}$ & Group 3<4<1,2 \\
Height $(\mathrm{cm})$ & $169.23 \pm 6.81$ & $171.88 \pm 6.18$ & $172.14 \pm 6.17$ & $171.40 \pm 6.71$ & $9.633^{* *}$ & Group $1<4<2<3$ \\
Weight $(\mathrm{kg})$ & $67.82 \pm 9.94$ & $71.18 \pm 9.84$ & $71.47 \pm 10.36$ & $70.03 \pm 10.63$ & $9.142^{* *}$ & Group $1,4<2,3$ \\
BMI $\left(\mathrm{kg} / \mathrm{m}^{2}\right)$ & $23.59 \pm 2.54$ & $24.03 \pm 2.63$ & $24.06 \pm 2.78$ & $23.76 \pm 2.83$ & $4.034^{* *}$ & Group $1,4<2,3$ \\
\hline
\end{tabular}

Values are presented as mean \pm standard deviation.

Group 1, vigorous physical activities for at least 6 days; group 2, vigorous physical activities for 3-5 days; group 3, vigorous physical activities for 1-2 days; group 4, no physical activity; BMl, body mass index.

${ }^{* *} P<0.01$.

Table 2. Analysis of variance results for blood lipid and blood glucose

\begin{tabular}{lrrrrrr}
\hline Variable & \multicolumn{1}{c}{ Group 1 } & Group 2 & Group 3 & Group 4 & F & Post hoc \\
\hline TC $(\mathrm{mg} / \mathrm{dL})$ & $194.30 \pm 33.39$ & $193.66 \pm 32.11$ & $193.82 \pm 32.20$ & $195.16 \pm 32.50$ & 0.657 & - \\
TG $(\mathrm{mg} / \mathrm{dL})$ & $106.21 \pm 54.33$ & $127.84 \pm 87.39$ & $135.67 \pm 96.21$ & $138.13 \pm 99.89$ & $7.716^{* *}$ & Group 1,2<3,4 \\
HDL-C (mg/dL) & $58.07 \pm 16.25$ & $54.95 \pm 14.12$ & $53.96 \pm 14.10$ & $53.28 \pm 14.06$ & $6.867^{* *}$ & Group 1,2>3.4 \\
LDL-C (mg/dL) & $114.98 \pm 29.47$ & $113.14 \pm 30.41$ & $112.72 \pm 30.84$ & $114.25 \pm 30.75$ & 0.948 & - \\
Glucose (mg/dL) & $94.87 \pm 11.20$ & $96.03 \pm 14.27$ & $95.65 \pm 13.99$ & $95.58 \pm 13.35$ & 0.668 & - \\
\hline
\end{tabular}

Values are presented as mean \pm standard deviation.

Group 1, vigorous physical activities for at least 6 days; group 2, vigorous physical activities for 3-5 days; group 3, vigorous physical activities for $1-2$ days; group 4, no physical activity; TC, total cholesterol; TG, triglyceride; HDL-C, high-density lipoprotein cholesterol; LDL-C, low-density lipoprotein cholesterol.

${ }^{* *} P<0.01$.

\section{Blood analysis method}

To analyze blood components, blood was collected from the forearm vein using disposable syringes after the subjects took a rest for 10-20 min. in comfortable positions after having the subjects eat with the same eating habits during ordinary days on the day before the collection and eat no food after dinner. Out of the blood collected from each subject, $5 \mathrm{~mL}$ was put into a plain tube and centrifuged once at 3,000 rpm for $10 \mathrm{~min}$ using a Combi 408 centrifugal separator (Hanil Science, Daejeon, Korea) to separate only the serum components. Cobas-c702 biochemical automatic analyzer (Roche Diagnostics, Indianapolis, IN, USA) was used for analysis of blood lipids, blood glucose and other components. The reagents used for the analysis of lipid, blood glucose and other components were reagents dedicated to Cobas $\mathrm{C}$ systems; TC reagent-Cholesterol Gen.2, TG reagent- Triglycerides, HDL-C Reagent HDL-Cholesterol Plus 3rd generation, LDL-C reagent LDL-Cholesterol Plus 2nd generation, and Glucose HK Gen.3 for blood glucose.

\section{Statistical analysis}

The means and standard deviations by group were obtained to analyze the effects of the participation in strenuous activities at different degrees on lipids, blood glucose, blood pressure, and pulmonary functions. One-way analysis of variance (ANOVA) were conducted using the four groups with different degrees of participation in strenuous activities as independent variables. Since statistical significance appeared, the least significant difference tests were conducted as post boc tests. The statistical significance level ( $\alpha$ ) was set to 0.05 .

\section{RESULTS}

In the present study, lipids mean total cholesterol (TC), HDL-C, LDL-C, and triglyceride (TG) and these lipids in blood collected after fasting for at least $9 \mathrm{hr}$ were analyzed. The TC concentrations were shown to be $194.30 \pm 33.39 \mathrm{mg} / \mathrm{dL}$ in group 1 (vigorous physical activities for at least 6 days), $193.66 \pm 32.11 \mathrm{mg} / \mathrm{dL}$ in group 2 (vigorous physical activities for 3-5 days), 193.82 \pm 32.20 $\mathrm{mg} / \mathrm{dL}$ in group 3 (vigorous physical activities for $1-2$ days), and $195.16 \pm 32.50 \mathrm{mg} / \mathrm{dL}$ in group 4 (no physical activity). These results of the one-way ANOVAs were not statistically significant. The TG concentrations were shown to be $106.21 \pm 54.33 \mathrm{mg} / \mathrm{dL}$ in group $1,127.84 \pm 87.39 \mathrm{mg} / \mathrm{dL}$ in group 2, 135.67 \pm 96.21 $\mathrm{mg} / \mathrm{dL}$ in group 3, and $138.13 \pm 99.89 \mathrm{mg} / \mathrm{dL}$ in group 4. These results of the one-way ANOVAs were statistically significant $(F=$ 7.716, $P<0.01$ ), and according to the results of post hoc tests, the concentrations in groups 1 and 2 were significantly lower than those in groups 3 and 4 . The HDL-C concentrations were shown 
to be $58.07 \pm 16.25 \mathrm{mg} / \mathrm{dL}$ in group $1,54.95 \pm 14.12 \mathrm{mg} / \mathrm{dL}$ in group 2, $53.96 \pm 14.10 \mathrm{mg} / \mathrm{dL}$ in group 3, and $53.28 \pm 14.06 \mathrm{mg} /$ $\mathrm{dL}$ in group 4 . These results of the one-way ANOVAs were statistically significant $(F=6.867, P<0.01)$, and according to the results of post hoc tests, the concentrations in groups 1 and 2 were significantly higher than those in groups 3 and 4 . The LDL-C concentrations were shown to be $114.98 \pm 29.47 \mathrm{mg} / \mathrm{dL}$ in group $1,113.14 \pm 30.41 \mathrm{mg} / \mathrm{dL}$ in group $2,112.72 \pm 30.84 \mathrm{mg} / \mathrm{dL}$ in group 3, and $114.25 \pm 30.75 \mathrm{mg} / \mathrm{dL}$ in group 4 . These results of the one-way ANOVAs were not statistically significant.

The blood glucose measured in the present study was fasting blood glucose, which should be measured after fasting for at least $8 \mathrm{hr}$ in principle. In the case of blood glucose, $94.87 \pm 11.20 \mathrm{mg} /$ $\mathrm{dL}$ in group $1,96.03 \pm 14.27 \mathrm{mg} / \mathrm{dL}$ in group 2 , group 3 was $95.65 \pm 13.99 \mathrm{mg} / \mathrm{dL}$, and the 4 group was $95.58 \pm 13.35 \mathrm{mg} / \mathrm{dL}$. The results of one-way ANOVA were not statistically significant. The results of this study were summarized in Table 2 .

\section{DISCUSSION}

The present study analyzed the effects of participation in vigorous physical activities on blood lipids and blood glucose with data from health examination survey for workers. Therefore, the results of analyses of those variables that were observed through the study are mainly discussed.

Among changes resulting from human body's physiological responses and adaptation to physical activities, those in TC and TG are closely related to arteriosclerosis and coronary artery diseases (Levy et al., 1984). TC is affected by various factors of exercise such as the intensities of aerobic exercise and resistance exercise, durations of exercise, and the frequency of exercise (Mann et al., 2014). The results of the present study showed no significant difference between the groups. The results as such are consistent with the results of previous studies conducted by Stein et al. (1990) and Toriola (1984) in which middle-aged men performed jogging for 12 weeks indicating that no clear change occurred in TC as well as the results of a study indicating that TC is not easily reduced by training and that even complex exercises including regular aerobic exercise do not significantly improve body composition or blood lipids (Durstine et al., 2002). Although a complex exercise program consisting of aerobic exercise and resistance exercise was implemented for 12 weeks with obese women, TC levels did not change.

A previous study, indicated that long-term training is necessary to improve TC but whether the subjects in the present study per- formed physical activities for long periods of time because the questionnaire used in the present had questions about physical activities for the last one week. A study conducted by Tak et al. (1992) reported that TC values showed some weak correlations with age, blood pressure, and the degree of obesity, and blood glucose but did not show any significant correlation with alcohol consumption, smoking amounts, eating habits, or exercise habits.

TG is mainly stored in the liver and the subcutaneous fat and formed in the adipose tissues and the liver. The TG stored in the adipose tissue is rapidly involved in energy metabolism when sugar is insufficient as an energy source during exercise. Gaesser and Rich (1984) reported that TG decreased when men aged in a range of 35- 49 years ran $62.7 \mathrm{~km}$ per week on average and Hyeon et al. (1991) reported that TG significantly decreased in middle-aged women after they jogged for 8 weeks at a $70 \%$ of $\mathrm{VO}_{2}$ intensity. TG significantly decreased after regular and continuous exercise.

HDL-C is known to be a preventative factor for coronary heart diseases as it binds them to LDL receptors in the arterial wall cell membranes to inhibiting the entry of LDL into cells (Gordon et al., 1989). The increase in HDL-C is attributed to the activation of lipoprotein hydrolytic enzymes in the plasma, which leads to the conversion of the components of chylomicrons and low-density lipoprotein into HDL-C and the suppression of hepatic TGs activity resulting in low catabolism of HDL-C. In a study on treadmill exercise conducted with 753 middle-aged men for 1 year and 7 months, Blair et al. (1989) reported that HDL-C significantly increased along with increases in treadmill exercise time. Choi et al. (1996) reported that when middle-aged women performed exercise at intensities of 70\%-85\% maximum heart rate three times in a week, HDL-C significantly increased, consistently with the results of the present study.

LDL-C accounts for about $70 \%$ of TC and is known as an important risk factor for cardiovascular diseases because its increase leads to increases in the incidence of cardiovascular diseases. The results of the present study did not show any significant difference in LDL-C between the groups. Weltman et al. (1987) reported that the value of LDL-C did not change after resistance exercise for 20 and 14 weeks, respectively. Hur (2008) also reported that aerobic exercise and resistance exercise did not significantly reduced LDL-C and also reported that there was no change in LDL-C thereby supporting the results of the present study.

Studies that observed the effects of exercise therapy to improve the conditions of disorders of lipid metabolism reported that although TC and TG decreased, the decreases were not statistically significant (Kim, 1997) or reported that TC significantly decre- 
ased. Given the results of a previous study indicating that dietary restrictions have more positive effects on TC, TG, LDL-C, HDL-C than exercise therapy and the results of another previous study indicating that blood cholesterol concentrations of women are greatly affected by age, body weight, physical activities, nutrition, and hormones, and exercise and diet therapy should be implemented simultaneously and more diverse studies are necessary.

Regular physical activities prevent diabetes and cardiovascular disease, improve the condition of insulin resistance, and improve blood glucose control ability (Bassuk and Manson, 2005). World Health Organization recommends moderate intensity exercise for at least $30 \mathrm{~min}$ to prevent diabetes mellitus. When a person without diabetes performs moderate-intensity aerobic exercise, the increase in the peripheral glucose uptake is balanced with the glucose production in the liver so that the blood glucose is not changed in general. High-intensity short-term exercise can lead to the elevation of blood catecholamines and increases in glucose production resulting in high blood glucose, which can last for 1-2 hr (Marliss and Vranic, 2002). Complex exercise that comprises both aerobic exercise and resistance exercise may be more effective than single exercise in controlling blood glucose. Increases in the muscle mass thanks to resistance exercise may increase glucose uptake and aerobic exercise may improve insulin action leading to increases in glucose uptake (Cuff et al., 2003; Sigal et al., 2007). Evans et al. (2005) reported that moderate intensity and high intensity aerobic exercises were effective in improving the condition of insulin resistance in patients with type 2 diabetes and the elderly. American Diabetes Association (ADH) actually recommends moderate intensity and high intensity exercises in its physical activity guidelines for diabetes prevention and therapeutic methods (ADA, 2012). However, in the present study, blood glucose was shown to be $94.87 \pm 11.20 \mathrm{mg} / \mathrm{dL}$ in group $1,96.03 \pm 14.27 \mathrm{mg} / \mathrm{dL}$ in group $2,95.65 \pm 13.99 \mathrm{mg} / \mathrm{dL}$ in group 3 , and $95.58 \pm 13.35 \mathrm{mg} / \mathrm{dL}$ in group 4 . Post-test results were not statistically significant. Park et al. (2013) reported that statistically or numerically negative results were shown in all of body mass index, blood pressure, blood glucose, and TC after drinking and smoking regardless of exercise. Ahn (2000) reported that when regular exercise was performed for 10 weeks, the TC, LDL-C, and blood glucose decreased a little but the decreases were not statistically significant. These results are consistent with the results of this study.

Based on the results of the present study, the following conclusions could be obtained. First, participation in vigorous physical activities for at least three days positively affected HDL-C and TG. Second, participation in vigorous physical activities did not affect blood glucose.

\section{CONFLICT OF INTEREST}

No potential conflict of interest relevant to this article was reported.

\section{REFERENCES}

Ahn MY. The effect on fat, TCHO, glucose, and TG of middle-aged women by regular exercise. Korean J Phys Educ 2000;39:351-358.

American College of Sports Medicine. ACSM's guidelines for exercise testing and prescription. 9th ed. Philadelphia (PA): Lippincott Williams \& Wilkins; 2014.

American Diabetes Association. Standards of medical care in diabetes-2012. Diabetes Care 2012;35 Suppl 1:S11-63.

Baker TT, Allen D, Lei KY, Willcox KK. Alterations in lipid and protein profiles of plasma lipoproteins in middle-aged men consequent to an aerobic exercise program. Metabolism 1986;35:1037-1043.

Bassuk SS, Manson JE. Epidemiological evidence for the role of physical activity in reducing risk of type 2 diabetes and cardiovascular disease. J Appl Physiol (1985) 2005;99:1193-1204.

Blair SN, Kohl HW 3rd, Paffenbarger RS Jr, Clark DG, Cooper KH, Gibbons LW. Physical fitness and all-cause mortality. A prospective study of healthy men and women. JAMA 1989;262:2395-2401.

Brownell KD, Bachorik PS, Ayerle RS. Changes in plasma lipid and lipoprotein levels in men and women after a program of moderate exercise. Circulation 1982;65:477-484.

Choi HN, Park KH, Joo MH. Effects of long-term aerobic exercise on plasma total-cholesterol, triglyceride, glucose and ventilatory functions. Exerc Sci 1996;5:25-35.

Colberg SR, Sigal RJ, Fernhall B, Regensteiner JG, Blissmer BJ, Rubin RR, Chasan-Taber L, Albright AL, Braun B; American College of Sports Medicine; American Diabetes Association. Exercise and type 2 diabetes: the American College of Sports Medicine and the American Diabetes Association: joint position statement. Diabetes Care 2010;33:e147167.

Cuff DJ, Meneilly GS, Martin A, Ignaszewski A, Tildesley HD, Frohlich JJ. Effective exercise modality to reduce insulin resistance in women with type 2 diabetes. Diabetes Care 2003;26:2977-2982.

Durstine JL, Grandjean PW, Cox CA, Thompson PD. Lipids, lipoproteins, and exercise. J Cardiopulm Rehabil 2002;22:385-398.

Evans EM, Racette SB, Peterson LR, Villareal DT, Greiwe JS, Holloszy JO. Aerobic power and insulin action improve in response to endurance exercise training in healthy 77-87 yr olds. J Appl Physiol (1985) 2005; 
98:40-45.

Gaesser GA, Rich RG. Effects of high- and low-intensity exercise training on aerobic capacity and blood lipids. Med Sci Sports Exerc 1984;16: 269-274.

Gordon DJ, Probstfield JL, Garrison RJ, Neaton JD, Castelli WP, Knoke JD, Jacobs DR Jr, Bangdiwala S, Tyroler HA. High-density lipoprotein cholesterol and cardiovascular disease. Four prospective American studies. Circulation 1989;79:8-15.

Hermansen L, Stensvold I. Production and removal of lactate during exercise in man. Acta Physiol Scand 1972;86:191-201.

Hur MD. The effects of aerobic exercise and resistance exercise on the pulmonary function and blood lipids on male college students. Korean J Sports Sci 2008;17:617-630.

Hyeon SJ. Yeo NH, Park JD. The exercise prescription for prevention of an adult's disease in middle-aged men. Korean J Sports Med 1991;9:185200.

Jeong JH, Choi YS, Kim SM, Choi SY, Kim JE, Kim EY, Kim EY, Lee HY, Jung JH, Jin R. Correlation between physical activity stauts dyslipidemia in Korean adults: The 2010 Korea National Health and Nutrition Examination Survey. Korean J Clin Geri 2014;15:35-44.

$\mathrm{Kim} \mathrm{CH}$. The effects of treadmill exercise training on cardiopulmonary function and blood glucose in patients with NIDDM. Korean J Sports Med 1997;15:28-35.

Kim KH, Kim BJ, Kim WS, Park DH, Park SJ, Oh SH, Jung TW, Cho MH, Han KH. Introduce of physical education. Seoul: Rainbow Books; 2011.

Lee HJ, Kang SJ, Ko IK, Kim KD, Kim KR, Kim KB, Kim KH, Kim KJ, Kim BS, Kim SE, Kim HC, Noh HT, Park KS. Health and wellness. 8th ed. Seoul: Yeoungmunsa; 2010.

Levy RI, Brensike JF, Epstein SE, Kelsey SF, Passamani ER, Richardson JM, Loh IK, Stone NJ, Aldrich RF, Battaglini JW. The influence of changes in lipid values induced by cholestyramine and diet on progression of coronary artery disease: results of NHLBI Type II Coronary Intervention Study. Circulation 1984;69:325-337.

Mann S, Beedie C, Jimenez A. Differential effects of aerobic exercise, resis- tance training and combined exercise modalities on cholesterol and the lipid profile: review, synthesis and recommendations. Sports Med 2014;44:211-221.

Marliss EB, Vranic M. Intense exercise has unique effects on both insulin release and its roles in glucoregulation: implications for diabetes. Diabetes 2002;51 Suppl 1:S271-283.

Park HC, Rhyu HS, Roh HT. The effects of exercise drinking, smoking on body mass index, blood pressure, blood glucose, and total cholesterol in middle-aged men. J Korean Soc Wellness 2013;8:181-189.

Rasmussen SS, Glümer C, Sandbaek A, Lauritzen T, Borch-Johnsen K. Determinants of progression from impaired fasting glucose and impaired glucose tolerance to diabetes in a high-risk screened population: 3 year follow-up in the ADDITION study, Denmark. Diabetologia 2008;51:249-257.

Sigal RJ, Kenny GP, Boulé NG, Wells GA, Prud'homme D, Fortier M, Reid RD, Tulloch H, Coyle D, Phillips P, Jennings A, Jaffey J. Effects of aerobic training, resistance training, or both on glycemic control in type 2 diabetes: a randomized trial. Ann Intern Med 2007;147:357-369.

Stein RA, Michielli DW, Glantz MD, Sardy H, Cohen A, Goldberg N, Brown CD. Effects of different exercise training intensities on lipoprotein cholesterol fractions in healthy middle-aged men. Am Heart J 1990;119(2 Pt 1):277-283.

Tak YJ, Yoo SM, Cho B, Song YM, Yoo T, Huh BY. Factors related to serum total cholesterol. J Korean Acad Fam Med 1992;13:935-942.

Toriola AL. Influence of 12-week jogging on body fat and serum lipids. Br J Sports Med 1984;18:13-17.

Twigg SM, Kamp MC, Davis TM, Neylon EK, Flack JR; Australian Diabetes Society; Australian Diabetes Educators Association. Prediabetes: a position statement from the Australian Diabetes Society and Australian Diabetes Educators Association. Med J Aust 2007;186:461-465.

Weltman A, Janney C, Rians CB, Strand K, Katch FI. The effects of hydraulic-resistance strength training on serum lipid levels in prepubertal boys. Am J Dis Child 1987;141:777-780. 